\title{
Comparative Evaluation of Apical Extrusion of Debris During Root Canal Preparation Using two Rotary Systems - An In vitro Study
}

\author{
Hanumanthu Kumari*, S Datta Prasad, C Sunil kumar, N Vamsee Krishna, S Sunil kumar and KS Chandra Babu \\ Department of Conservative Dentistry and Endodontics, C. K. S. Teja Institute of Dental Sciences and Hospital, India \\ *Corresponding Author: Hanumanthu Kumari, Department of Conservative Dentistry and Endodontics, C. K. S. Teja Institute of Dental \\ Sciences and Hospital, India
}

Received: November 11, 2019; Published: November 27, 2019

DOI: $10.31080 /$ ASDS.2019.03.0715

\begin{abstract}
Aim and Objective: This in vitro study was aimed to evaluate the amount of apically extruded debris following root canal preparation using two different instrumentation systems.

Materials and Methodology: Twenty Extracted single rooted human teeth were included in this study. These samples were divided into 2 Groups. Two different Ni Ti rotary file systems wave one gold and one curve file were used. Canal patency was established by placing 20 size K-file up to the apical foramen and working length was determined by visual method where an ISO 20k file was placed in to the root canal until the tip of the file was visible at apical foramen and $1 \mathrm{~mm}$ subtracted from the obtained length. After that these samples were divided into 2 groups Group1: Wave one gold, Group 2: one curve file. After that instrumentation is done according to the manufacturer's instruction. Extruded debris and irritant extruded during instrumentation were collected into reweighed Eppendorf tubes. Then the tubes were stored in an incubator at $70^{\circ} \mathrm{C}$ for 5 days. The Eppendorf tubes were weighed to obtain the final weight of the extruded debris.

Results: There were statistically significant differences among the groups. The Wave One reciprocating system showed the more amount of apical extruded debris than that of one curve file $(P>0.05)$.

Conclusion: All instrumentation techniques were associated with debris and irritant extrusion but Wave one gold exhibit greater extrusion compared to One curve file.

Keywords: Apical Extrusion; Debris; Irritants; One Curve File; Wave One Gold Single File Reciprocating System; Incubator; Electrical Balance
\end{abstract}

\section{Introduction}

Complete disinfection of the root canal system and absolute three-dimensional obturation is the main goal of endodontic instrumentation [1]. According to Grove, thorough instrumentation in apical third is an essential requisite in cleaning and shaping of root canal [2]. To attain these goals, during the root canal preparation, debris such as necrotic pulp tissue, dentinal shavings, bacteria and their by-products or irritants may get released into the periarticular tissues which can lead to an inflammatory response, postoperative pain and possible delayed healing [3]. At present, all preparation techniques and instruments produce apical debris extrusion. Manual instrumentation happens to produce more apical extrusion of debris than that of engine driven rotary motion. Vande Visse and Brilliant have shown that in the absence of irritant, there is no extrusion of debris was observed while a thick worm of debris extruded when an irritant was used [4]. Recently advanced instrument designs such as radial lands, different flute depth, non cutting tips, variable tapers and cross-sections and different operating principles has been developed to improve working safety, and to shorten the working time and to create a greater flare within the preparations. These newer systems also provide a cleaner and smoother preparation to achieve the final obturation [5]. Most common finding of some studies examining the amount of apically extruded debris and irritant was that the instrumentation techniques which use a push and pull motion tends to produce a more apical extrusion of debris than instrumentation techniques using a rotational motion.

The purpose of this invitro study was to compare and evaluate the amount of apically extruded debris by two different rotary instruments using one curve file and Wave One gold. 


\section{Methodology}

Twenty freshly Extracted single rooted teeth with single root canals were collected. Initial debridement was done to remove the calculi and soft tissue remnants on the external surface with a periodontal curette. Inclusion criteria - patent single root canal, Fully formed apices. Exclusion criteria - calcifications, Resorption, Complicated root canal morphology. Standard access cavity was prepared, the pulp tissue was removed prior to make sure that chemo mechanical preparation process extrudes the dentinal shavings but not the pulpal remnants. Incisal edges were flattened by using high speed bur and considered as reference point. Size $20 \mathrm{~K}$ file (Mani K File) was used to establish the canal patency. Working length was established by visual method with an ISO $20 \mathrm{~K}$ file is placed in the root canal until the tip of the file was visible at apical foramen and $1 \mathrm{~mm}$ was subtracted from obtained length. According to the file used for the biomechanical preparation, the teeth were randomly divided into two groups.

Group 1: Wave one gold (Dentsply Maillefer, Ballaigues, Switzerland)

Reciprocating back and forth motion was used with a reciprocating wave one gold file. According to the manufacturer's instructions a size 25 reciprocating wave one gold file with 0.07 taper was used in slow in and out pecking motion. After every three pecks the flutes of the instrument were cleaned. As the initial size of all the canals was equal to size 20 no glide path was created before instrumentation. At a maximum torque level of $2.5 \mathrm{~N} / \mathrm{cm}$ the instruments were used at a speed of 350RPM6.

\section{Group 2: One curve file (Micro Mega, Besancon, France)}

Without applying any pressure the one curve rotary file was used in and out motion. With tip size of 25 and 0.06 taper these instruments were used at a speed of 300RPM according to the manufacturer's instructions with a torque of $2.5 \mathrm{~N} / \mathrm{cm} \mathrm{[6].}$

The experimental model described by Myers and Montgomery method was used in this study [7]. The Eppendorf tubes with separated stoppers were used in this study. The electrical analytical balance $10-4 \mathrm{~g}$ was used to measure the initial weights of the Eppendorf tubes. The mean value for all the three consecutive weights for each tube was calculated. On Each stopper, a hole was created and each tooth was cemented up to CEJ and open ended 25 -G needle was placed along side of the stopper and it acting as a drainage canula to maintains the pressure inside and outside of the tube. The stopper with the tooth and the needle was attached to the Eppendorf tube. These tubes were fitted into vials. Between the files in each sample the irritant used was $10 \mathrm{ml}$ distilled water. From the vial after instrumentation the Eppendorf tube was removed immediately. The stopper, needle and the tooth were separated from the tube after the completion of chemo mechanical preparation. $1 \mathrm{ml}$ of distilled water was used to wash the root and collect the debris adhered to the root surface into the tube. After that these samples kept in a incubator at $70^{\circ} \mathrm{C}$ for 5 days to evaporate distilled water before obtaining the weight of the dried debris. Final weight of the tubes with extruded debris was obtained by using electrical analytical balance. The mean value of the three consecutive weights of each tube was calculated. By subtracting the weight of the empty tubes from that of the tubes containing debris the dry weight of the extruded debris was calculated.

\section{Results}

\section{Statistical analysis}

The data was analysed with Statistical Package for Social Sciences (SPSS) for Windows 20.0 (SPSS, Inc. chicago, Illinois). The descriptive data shows mean, standard deviation (SD) and standard error (SE) for all the groups separately and were used for comparison between the groups. The observed data was analysed by Mawhinney's u test to compare the mean weight of debris among the groups wave one gold and one curve. Confidence intervals were set at $95 \%$ and values of $\mathrm{p}<0.05$ were interpreted as statistically significant.

\begin{tabular}{|l|c|c|c|c|c|c|}
\hline Group & $\mathbf{N}$ & $\begin{array}{c}\text { Mean } \\
\text { Weight of } \\
\text { Debris }\end{array}$ & $\begin{array}{c}\text { Std. } \\
\text { Deviation }\end{array}$ & $\begin{array}{c}\text { Std. Error } \\
\text { Mean }\end{array}$ & $\begin{array}{c}\mathbf{U} \\
\text { statistic }\end{array}$ & $\begin{array}{c}\mathbf{P} \\
\text { value }\end{array}$ \\
\cline { 1 - 5 } $\begin{array}{l}\text { Wave } \\
\text { one } \\
\text { gold }\end{array}$ & 10 & .021710 & .0128525 & .0040643 & 20 & 0.023 \\
\cline { 1 - 4 } $\begin{array}{l}\text { One } \\
\text { curve }\end{array}$ & 10 & .010690 & .0038382 & .0012138 & & \\
\hline
\end{tabular}

Table 1: Interpretation: weight of extruded debris is higher in wave one gold and difference is statistically significant $(p=0.023$ i. $e<0.05)$.

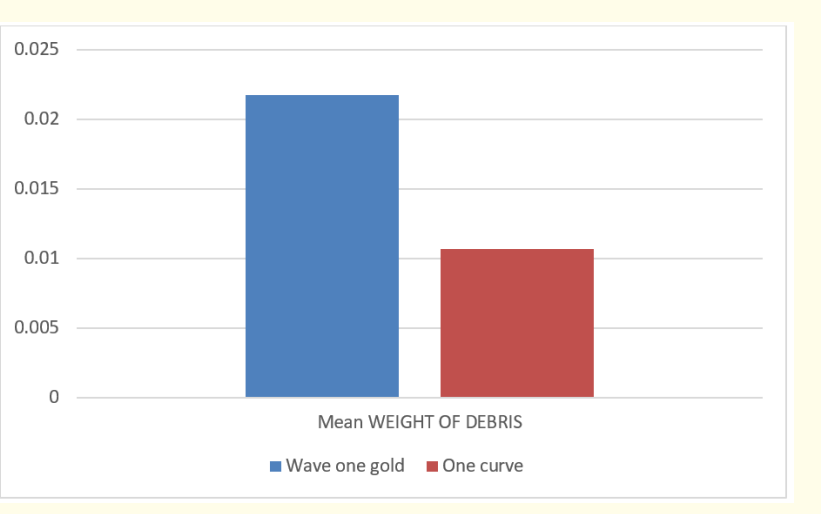

Figure 1 


\section{Discussion}

In endodontic treatment most common reason for mid treatment flare-up is extrusion of debris into the periarticular tissue. All samples were of $19 \mathrm{~mm}$ length and apical file size was also same in this study. The important phase of cleansing the canal is irrigation. In this study distilled water was used an irritant because it prevents additional weight increase due to $\mathrm{Na}$ OCl crystal formation. The quantity of irritant used in this study was $10 \mathrm{ml}$ of distilled water for each sample, working length for all samples was $1 \mathrm{~mm}$ short of apical foramen. Many studies have shown that when instrumentation was performed to the apical foramen, results more debris was forced apically so that variables which may affect results could be minimized. Martin and Cunningham demonstrated greater debris extrusion when canals were instrumented at a length where the file was observed to just protrude through the apical foramen versus $1 \mathrm{~mm}$ short of apical foramen [8]. Myers and Montgomery clearly showed that the working length $1 \mathrm{~mm}$ short of canal length contributed to significantly less debris extrusion [7]. Two different rotary (one curve file) and reciprocating single file system (wave one gold) was used to compare the apical extrusion of debris. The results reveal that the extrusion of debris occurs independent of the motion or design of the instrument. One curve file extrudes less debris than that of wave one gold file.

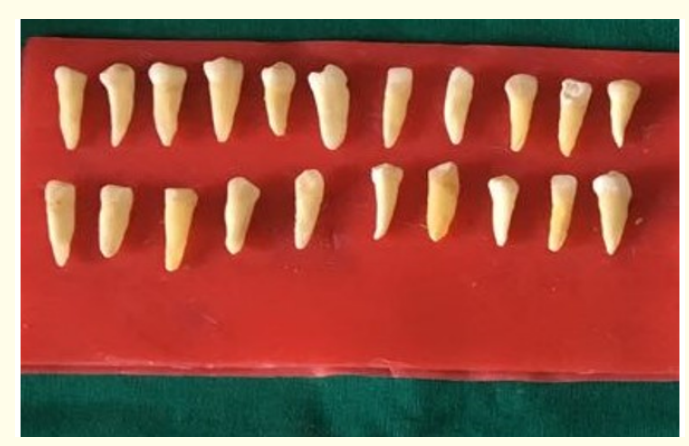

Figure 2: Extracted single rooted human teeth.

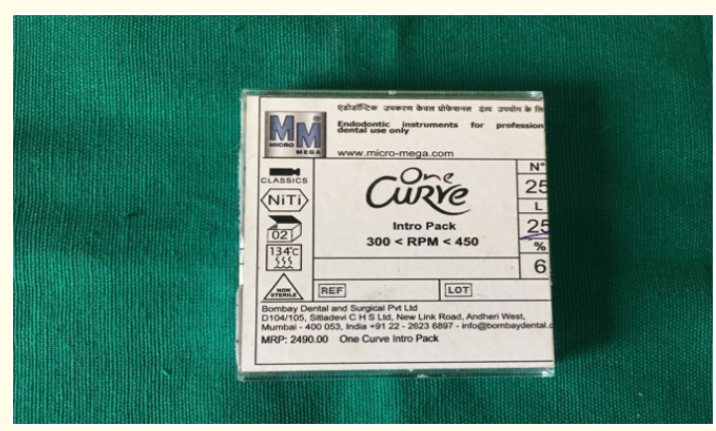

Figure 3: One curve rotary file, Wave one gold reciprocating file.

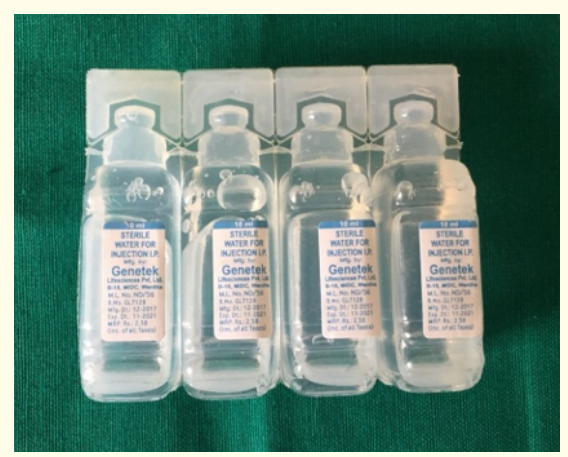

Figure 4: Distilled water.

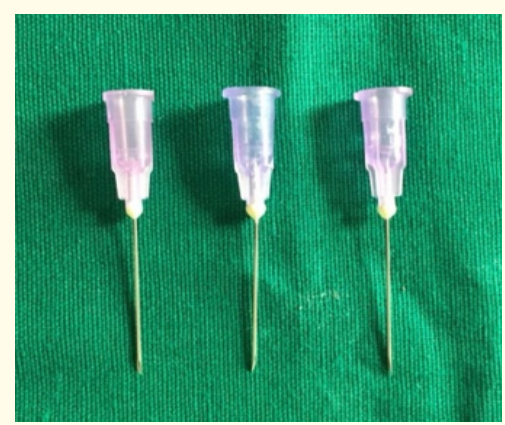

Figure 5: 25 gauge needle.

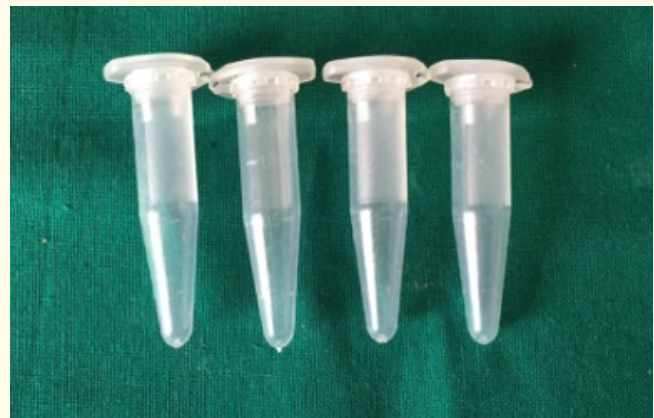

Figure 6: Eppendorf tubes.

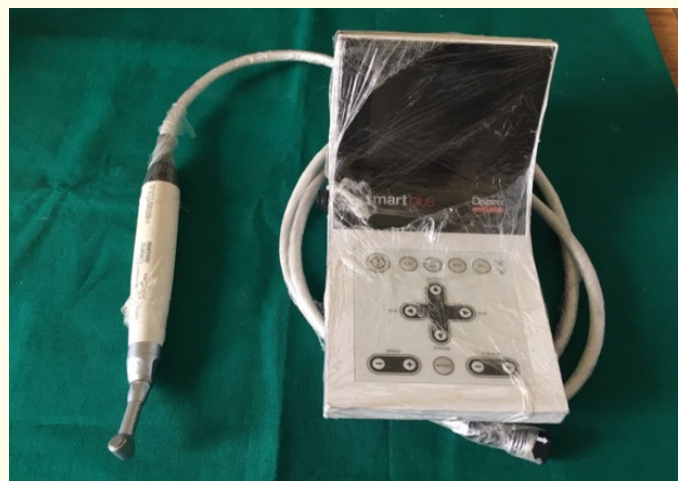

Figure 7: X smart plus endodontic motor. 


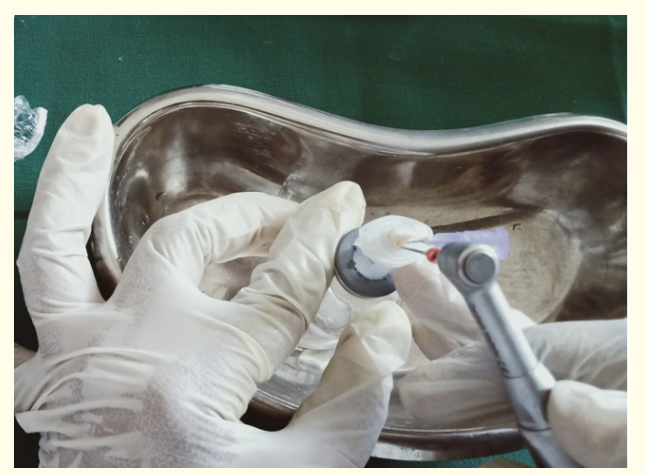

Figure 8: Instrumentation with wave one gold file.

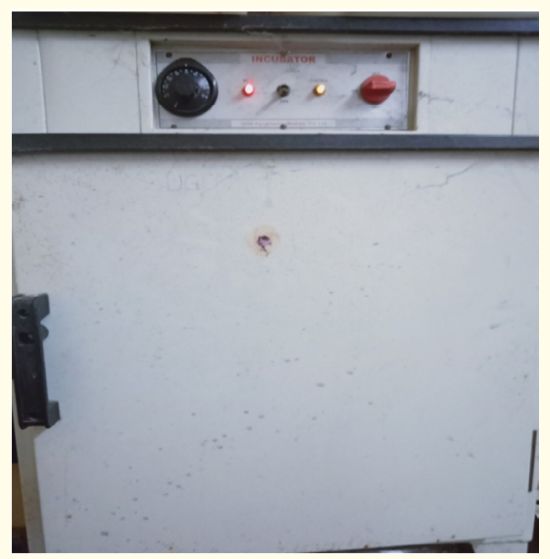

Figure 9: Incubator.

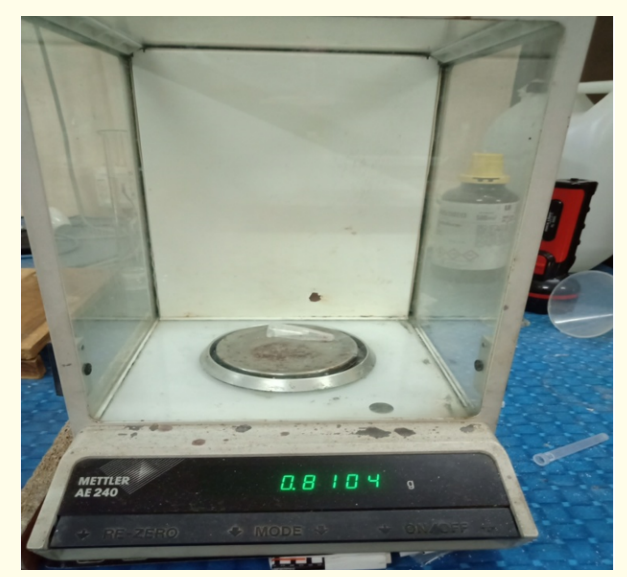

Figure 10: Electronic microbalance.

One curve file was introduced by Micro Mega, Besancon, France. It is the $\mathrm{Ni} \mathrm{Ti} \mathrm{instrument} \mathrm{used} \mathrm{in} \mathrm{continuous} \mathrm{rotation} \mathrm{for} \mathrm{quality}$ root canal treatment. One shape file is a single instrument used in full clockwise rotation. Its tip size of 25 and constant taper of 0.06 and different cross sectional designs present over the complete length of the working part [3]. symmetrical cutting edges at the apical part. The number of cutting edges decreases to two in the middle part, this part is asymmetrical. There are $2 \mathrm{~S}$ shaped cutting edges in the coronal part. Non cutting safety tip provides an effective apical progression avoiding obstructions which are often preceded by instrument separation. Speed for one shape is $300 \mathrm{rpm}$, Torque $2.5 \mathrm{~N} / \mathrm{cm}$ as recommended by manufacturer [9].

Wave one gold instruments employs thermal process, give a super elastic $\mathrm{Ni}$ Ti file. It achieves flexibility and strength. It utilizes single file and single use hypothesis. Wave One Gold file has a parallelogram cross section with two 85 degree cutting edges in contact with the canal wall and $24^{\circ}$ helical angle present at the active part of the file. The extra space around the instrument also gives space for debris removal. Roundly tapered and semi-active tip present in wave one gold [10] It cuts dentin by engaging in a $150^{\circ} \mathrm{CCW}$ direction and then disengage $30^{\circ}$ in a CW direction. Therefore, the instrument makes a reverse rotation of $360^{\circ}$ after three cycles [10].

Wave one gold removes radicular dentin in short period of time by its aggressive ability of cutting action but unable to, displace debris coronally that increase apical extrusion in combination with reciprocal motion. The reciprocation movement in Wave One gold system is formed by a wider cutting angle and smaller release angle. While rotating in the release angle the flutes will not remove debris but push them apically. Moreover, Wave One gold file is quite big, rigid with an increased taper (in the study 0.07 taper primary file is used which corresponds to ISO size 25) which is directed to reach the apex. Moreover, instrumentation in reciprocal motion may enhance debris transportation toward the apex. Besides instrumentation in reciprocal action may increase transportation of debris in the direction of the apex [11]. For this reason continuous rotary motion acts like a screw conveyor and increase the transportation of dentin debris in coronal direction. Due to presence of changeable cross section all along blade of the file provides cantering ability in apical 3rd and superior debris removal up to medium and coronal parts [12].

\section{Conclusion}

Based on results of the study, it can be concluded that all instrumentation systems even full sequence continuous rotary or reciprocating caused apical debris extrusion. There was significant difference in debris extrusion between one curve file and wave one gold rotary file system. One curve full sequence rotary file system was associated with significantly less debris extrusion compared with wave one gold reciprocating file system. 


\section{Bibliography}

1. Logani Ajay and Naseem Shah. "Apically extruded debris with three contemporary Ni-Ti instrumentation systems: An ex vivo comparative study". Indian Journal of Dental Research 19.3 (2008): 182.

2. Grove CJ. "The value of the dentinocemental junction in pulp canal surgery". Journal of Dental Research 11 (1931): 466-468.

3. Singh Abhishek., et al. "Apical extrusion of debris and irrigants using ProTaper hand, M-two rotary and WaveOne single file reciprocating system: An ex vivo study". Journal of Conservative Dentistry: JCD 18.5 (2015): 405.

4. VandeVisse Jack E and J David Brilliant. "Effect of irrigation on the production of extruded material at the root apex during instrumentation". Journal of Endodontics 1.7 (1975): 243-246.

5. Hegde Mithra N and S. Thatte. "Comparison of the amount of apical extrusion of bacteria following the use of different instrumentation techniques-An in vitro study". Nitte University Journal of Health Science 1 (2011): 27-32.

6. Dincer., et al. "Apical extrusion of debris during root canal preparation using a novel nickel-titanium file system: WaveOne gold". Journal of Conservative Dentistry: JCD 20.5 (2017): 322.

7. Myers Garry L and Steve Montgomery. "A comparison of weights of debris extruded apically by conventional filing and Canal Master techniques". Journal of Endodontics 17.6 (1991): 275-279.

8. Martin Howard and Walter T Cunningham. "The effect of endosonic and hand manipulation on the amount of root canal material extruded". Oral Surgery, Oral Medicine, Oral Pathology 53.6 (1982): 611-613.

9. Singbal Kiran., et al. "Comparative evaluation of apically extruded debris during root canal instrumentation using two Ni-Ti single file rotary systems: An in vitro study." Journal of Conservative Dentistry: JCD 20.2 (2017): 64.

10. Dincer., et al. "Apical extrusion of debris during root canal preparation using a novel nickel-titanium file system: WaveOne gold". Journal of Conservative Dentistry JCD 20.5 (2017): 322.

11. Hadi Ali W and Mohammed R Hameed. "Comparative Study of The Amount of Apically Extrusion of Debris During Root Canal Preparation Using Wave One ${ }^{\mathrm{TM}}$, Trushape 3D $\mathrm{D}^{\mathrm{TM}}$, Hyflex ${ }^{\mathrm{TM}}$ $\mathrm{CM}$ and One Shape ${ }^{\mathrm{TM}}$ Instrumentation Systems (An In Vitro Study)". Journal of Baghdad College of Dentistry 29.1 (2017): 1-8. 322.
12. Nevares Giselle., et al. "Apical extrusion of debris produced during continuous rotating and reciprocating motion". The Scientific World Journal (2015).

Volume 3 Issue 12 December 2019

(C) All rights are reserved by Hanumanthu Kumari., et al. 